\title{
Investigation of the coupling properties of the ion cyclotron fast wave under applied magnetic perturbations and MHD phenomena in ASDEX Upgrade
}

\author{
Guillermo Suárez López ${ }^{1,2, \star}$, Roman Ochoukov ${ }^{1}$, Matthias Willensdorfer ${ }^{1}$, Volodymyr Bobkov ${ }^{1}$, Mike Dunne ${ }^{1}$, Helmut \\ Faugel $^{1}$, Helmut Fünfgelder ${ }^{1}$, Jean-Marie Noterdaeme ${ }^{1,3}$, Erika Strumberger ${ }^{1}$, Wolfgang Suttrop ${ }^{1}$, Hartmut Zohm $^{1,2}$, \\ the ASDEX Upgrade team, and the EUROfusion MST1 Team ${ }^{\star \star}$ \\ ${ }^{1}$ Max Planck Institute for Plasmaphysics, Boltzmannstraße 2, 85748 Garching bei München, Germany \\ ${ }^{2}$ Ludwig Maximillian University of Munich, Geschwister-Scholl-Platz 1, 80539 München, Germany \\ ${ }^{3}$ Applied Physics Department, University of Ghent, Sint-Pietersnieuwstraat 41, 9000 Ghent, Belgium
}

\begin{abstract}
The modulation of the ion cyclotron fast wave coupling to the plasma due to non-axisymmetric changes of the distance antenna-R-cutoff is studied. These changes can arise when magnetic perturbation (MP) fields are used, or when MHD activity is present. The application of MP fields can excite a low field side midplane plasma kink response that amplifies the vacuum perturbation field, leading to appreciable 3D plasma displacements. This effect is studied via NEMEC simulations. Rigid rotation of the MP field is found to produce a coherent antenna loading resistance modulation, suggesting an interplay between the non-axisymmetric magnetic field structure and the wave coupling properties. MHD modes are shown to introduce similar loading resistance oscillations, coherent with the mode rotation frequency. The case of a $(2,1)$ mode is presented.
\end{abstract}

\section{Introduction}

The excitation of the fast magnetosonic wave in the ion cyclotron range of frequencies (few tens of $\mathrm{MHz}$ ) is an effective technique to transport several megawatt of power up to the core plasma of a fusion device. Its excitation is produced by launchers, usually, antennas that are flushed with the first wall of the plasma device, such as a tokamak [1], and the wave energy is deposited via damping mainly into the ion species.

The major drawback of the technique is the fact that the launched fast wave with $k_{\|} \neq 0$ is evanescent in lowdensity plasmas, such as the ones encountered in the socalled scrape-off layer or edge plasma region of a tokamak. The transported power into the non-evanescent, higher density, plasma can then be expressed as an exponential decay that depends on the optical thickness $(\eta)$ of the evanescent region, i.e. $P_{\text {trans }} \approx P_{0} e^{-2 \eta}$ with $\eta=$ $k_{0} \int_{l} n(l) d l$. For a monotonically increasing perpendicular refractive index $n_{\perp, F W}^{2} \in\left[n_{0 \perp, F W}^{2}, 0\right]$, the transported power can be approximated as $P_{\text {trans }} \approx P_{0} e^{-1.1 k_{\|} d}$ [2] with d being the distance from the antenna up to the R-cutoff where the wave becomes propagative, i.e. $n_{\perp, F W}^{2}>0$.

In this study, we address non-axisymmetric changes of the distance $« d »$ arising from the application of magnetic perturbation (MP) fields from in-vessel saddle coils and naturally occurring MHD modes. Measurements of the antenna performance via loading resistance are used in order to assess their effect on the accessibility of the fast wave to the propagating region. Furthermore, simulations are performed with the ideal non-linear MHD code
NEMEC (NESTOR code for the free boundary problem + VMEC code) [3] to predict the plasma kink response under different applied MP phasing.

The paper is organized as follows: In section 2 the experimental setup is described, including a brief description of the ASDEX Upgrade saddle coils and the ion cyclotron system. In section 3 the analyzed discharges are presented and NEMEC predictions are shown. In section 4 the influence of MHD modes on the loading resistance of the antennas is described for the specific case of a $(2,1)$ mode. Finally, in section 5 conclusions are drawn and a future outlook is sketched.

\section{Experimental setup}

\subsection{Magnetic perturbation saddle coils}

The ASDEX Upgrade tokamak is complemented with two poloidally shifted toroidally-distributed arrays of eight invessel saddle coils. MP fields are applied from these coils for edge localized mode (ELM) mitigation and suppression studies, mode unlocking, and resistive wall mode control [4]. Individual power sources feeding each coil allow a wide range of configurations. The coil currents can be set to $0<I_{\text {coil }}<1.25 \mathrm{kA} /$ turn and the phasing between the upper toroidal row and lower row can be set to $-\pi<\Delta \varphi_{U L}<\pi$. The applied phase difference determines the vacuum poloidal spectrum of the MP field.

\footnotetext{
*e-mail: guillermo.suarez@ipp.mpg.de

${ }^{\star \star}$ See the author list of IAEA FEC 2016 OV/P-12 by H. Meyer et al. , to be published in Nuclear Fusion
} 
When the MP field is applied, a plasma response is commonly observed, especially strong at low collisionality $\left(v^{*}\right)$, that leads to pressure driven kink modes around the low field side (LFS) midplane and a peeling response around the X-Point and plasma top [5, 6]. Both responses are sensitive to the applied MP field poloidal and toroidal spectrum. Furthermore, the plasma response can amplify the vacuum MP field, leading to non-axisymmetric displacements that range from a few millimeters to the centimeter order [7], as illustrated in figure 1.

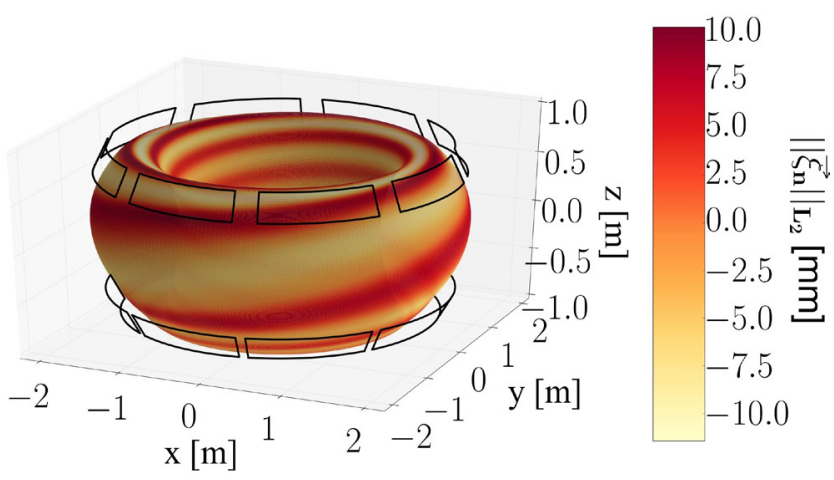

Figure 1. NEMEC 3D equilibrium of shot \#32091 at $\mathrm{t}=2.9 \mathrm{~s}$ where the saddle coils have been artificially supplied with 5kAt and $\Delta \varphi_{U L}=-140^{\circ}$ for this simulation. The normal displacement of the last closed flux surface $\left\|\vec{\xi}_{n}\right\|_{L_{2}}$ is color-coded.

\subsection{Ion cyclotron resonance heating (ICRH) system and diagnostics}

Four ICRH antennas are presently installed in ASDEX Upgrade: a pair of 2-strap antennas with boron coated limiters and a pair of 3-strap antennas with tungsten coated limiters [8]. The antennas are fed in pairs; the 2-strap antennas (a1 and a3) are fed by two RF generators connected together through two hybrid $3 \mathrm{~dB}$ couplers. The transmission lines are then individually matched by a set of two stubs and a T-junction. The 3-strap antennas (a2 and a4) are also fed by two RF generators, one feeds the central strap of each antenna and the other feeds the side straps via suitable $3 \mathrm{~dB}$ hybrid couplers. In this case, each individual transmission line is matched by two stubs without T-junction, see figure 2 .

The measured loading resistance $\left(R_{L}\right)$ is an experimentally accessible quantity that gives a good estimate of the coupled electromagnetic power to the plasma. It relates the power flowing through the coaxial line feeding a strap and the voltage maximum in that line, such that:

$$
R_{L}=\frac{2 P_{\text {line }} Z_{0}^{2}}{V_{\text {line, } \text { max }}^{2}}
$$

Where $Z_{0}$ is the characteristic impedance of the line. Behind the matching system, a directional coupler measures the forward and reflected power, thus providing $P_{\text {line }}$. A voltage probe located at the voltage antinode of the resonant line provides $V_{\text {line, } \max }$.

\section{Experimental results. NEMEC simulations}

\subsection{Analyzed discharges}

The three ASDEX Upgrade presented discharges, \#32319, \#32320 and \#32321 were performed at a toroidal magnetic field of $B_{t}=-2 \mathrm{~T}$ and a plasma current of $I_{p}=0.8$ MA, resulting in an edge safety factor of $q_{95} \approx-4.3$. Rigid rotations of the MP fields with different phasing at $\Delta \varphi_{U L}=-30^{\circ}, 40^{\circ}, 150^{\circ}$ respectively were performed with a maximum current of $I_{\text {coil }}=6.25 \mathrm{kAt}$ at $v=6 \mathrm{~Hz}$. The applied MP field is affected by the passive stabilization loop (PSL), a copper bulk holding the saddle coils that serves to increase the resistive time constant of the vertical instability. The PSL is induced with eddy currents whenever an AC current circulates the saddle coils, attenuating and lagging the MP field with respect to the applied coil current [9]. This results in an effective smaller MP field. At a frequency of $v=6 \mathrm{~Hz}$, the attenuation can become as high as $50 \%$.

The four ICRH antennas were switched on simultaneously with ranging powers, such that $0.6 \mathrm{MW}<P_{\text {antenna }}<$ $0.85 \mathrm{MW}$. The presented loading resistance time traces of figure 3 are chosen so discharge profiles, such as the normalized plasma beta $\beta_{N}$, line-averaged density $\left\langle n_{e}\right\rangle$, ICRH power $P_{I C R H}, q_{95}$, etc, were kept as similar as possible during the rigid rotation. A clear modulation of the loading resistance, coherent with the rotating MP field can be observed. It is seen that for the case of $\Delta \varphi_{U L}=+150^{\circ}$ the loading resistance changes as much as $\Delta R_{L} \sim 0.3 \Omega$, value peak to peak. This loading variation accounts for $\sim 20 \%$ of the base value, whereas for the case of $\Delta \varphi_{U L}=-30^{\circ}$ the change in loading is moderated $(\sim 10 \%)$. The case of $\Delta \varphi_{U L}=40^{\circ}$ presents a loading resistance variation of about $(\sim 10 \%-15 \%)$. This points to a dependence of the loading resistance variation with the applied MP phasing. The three discharges ended prematurely due to MHD activity deriving in a locked mode, hence difficulting a more profound analysis.

\subsection{NEMEC simulations}

A series of simulations were performed with the nonlinear spectral ideal MHD code NEMEC. NEMEC uses a variational principle to self-consistently minimize the plasma energy in the confined region, while the plasma boundary is deformed according to the external vacuum field (outside of the last closed flux surface) region. The saddle coils, as well as the other conductors (TF, PF and auxiliary coils), provide such vacuum field. The scrape-off layer is not included in the code due to its spectral description. Because of the lack of resistivity terms, the nested flux surfaces are preserved and the normal component of the magnetic field vector $\vec{B} \cdot \vec{u}_{n}$ vanishes. Hence, no magnetic islands can arise with the NEMEC code.

The experimental plasma pressure and toroidal current reconstructed from the CLISTE equilibrium code [10] serve as input to the NEMEC code. Plasma parameters from discharge \#32091 at 2.9 s were provided 

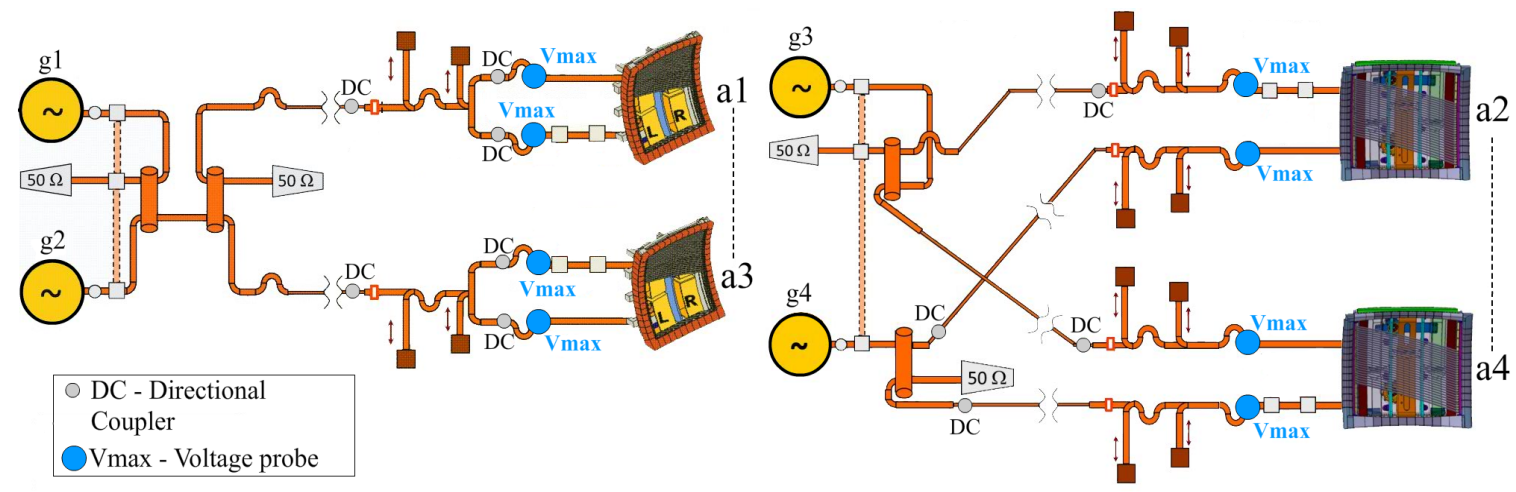

Figure 2. ASDEX Upgrade ICRH system. RF generators are marked from g1 to g4. Antenna 1 (a1) and antenna 3 (a3) are 2-strap antennas, antenna 2 (a2) and antenna 4 (a4) are 3-strap antennas.
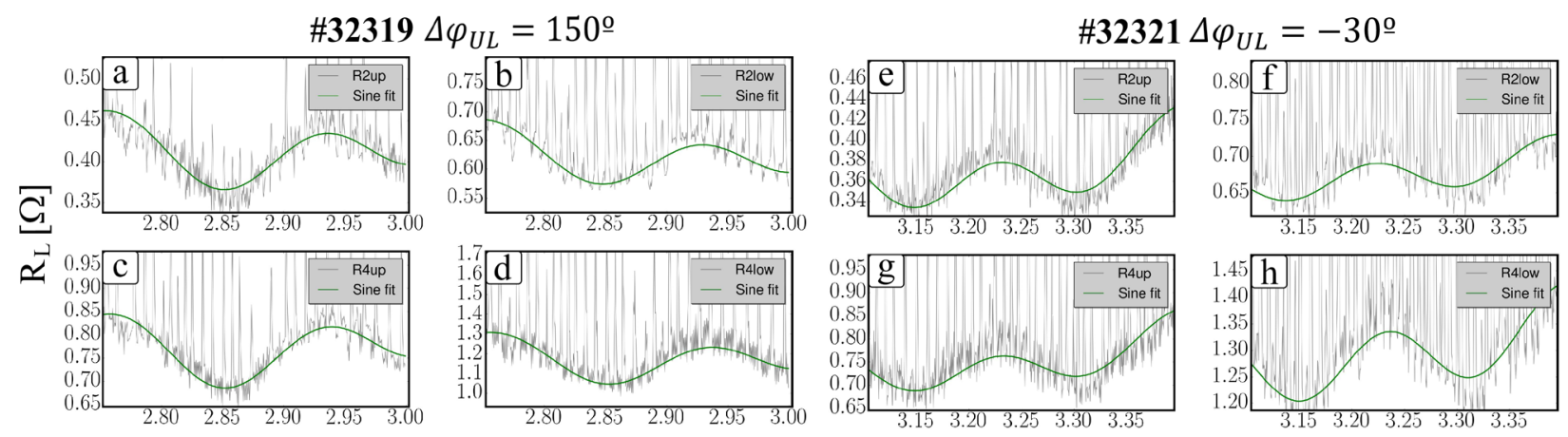

time $[\mathrm{s}]$

Figure 3. Loading resistances of the 3-strap antennas feeding lines in discharges \#32319 (a-d) and \#32321 (e-h). R2up designates a2, upper feeder and so on. A sine fit (green) is added to better visualize the base variation of the loading.

for the simulations. Discharge \#32091 is very close in parameters to the presented discharges where $R_{L}$ measurements have been taken, $q_{95}=-4.4, \beta_{N}=1.9$, $\left\langle n_{e}\right\rangle_{\text {edge }}=1.95 * 10^{19} \mathrm{~m}^{-3},\left\langle n_{e}\right\rangle_{\text {core }}=3.6 * 10^{19} \mathrm{~m}^{-3}$. The 2D converged NEMEC equilibrium was compared with the CLISTE one and minimal adjustment to the external PF coil currents were performed to match the q-profile from both codes. Then, the 3D free boundary NEMEC code was run, with the previously converged 2D equilibrium parameters, adding this time the different $\Delta \varphi_{U L}$ saddle coil cases in order to obtain the final nonaxisymmetric equilibria. The simulation parameters were chosen as 1000 flux surfaces, 26 poloidal mode numbers and 8 toroidal mode numbers for each period. For the saddle coils, $\mathrm{n}=2$ perturbations with $I_{\text {coil }}=5 \mathrm{kAt}$ were used. The LFS normal displacement at $\theta=0$ (midplane) of the computed last closed flux surface $\left(\left\|\vec{\xi}_{n}\right\|_{L_{2}}\right)$ is presented in figure 4 . The normal displacement is defined as the $L^{2}$ norm of the normal displacement vector to the axisymmetric last closed flux surface.

It is predicted from the simulations that a LFS midplane normal displacement of about $0 \mathrm{~mm}<\left\|\vec{\xi}_{n}\right\|_{L_{2}}<7$ mm should occur for different applied $\Delta \varphi_{U L}$ and $I_{\text {coil }}=5$ kAt. The ion cyclotron fast wave coupling to the plasma is very sensitive to the density profile in front of the launch-

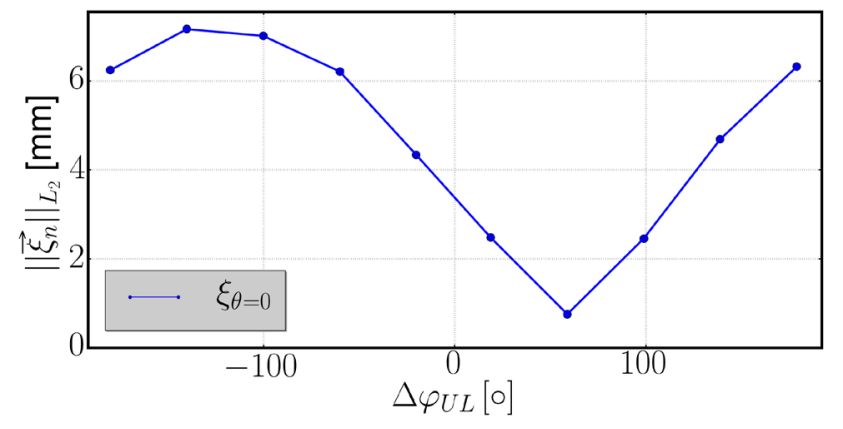

Figure 4. NEMEC LFS midplane normal displacement vector norm vs. different $\Delta \varphi_{U L}$.

ing antenna. A LFS midplane normal displacement of a few mm should influence this coupling. This phenomenon is indeed registered in the loading resistance measurements of figure 3, as a harmonic oscillation coherent with the rotating MP field. It is hence also expected from these simulations that the loading resistance variation should exhibit a $\Delta \varphi_{U L}$ dependence. A detailed comparison of loading resistance and density measurements with simulations is beyond the currently available data. 

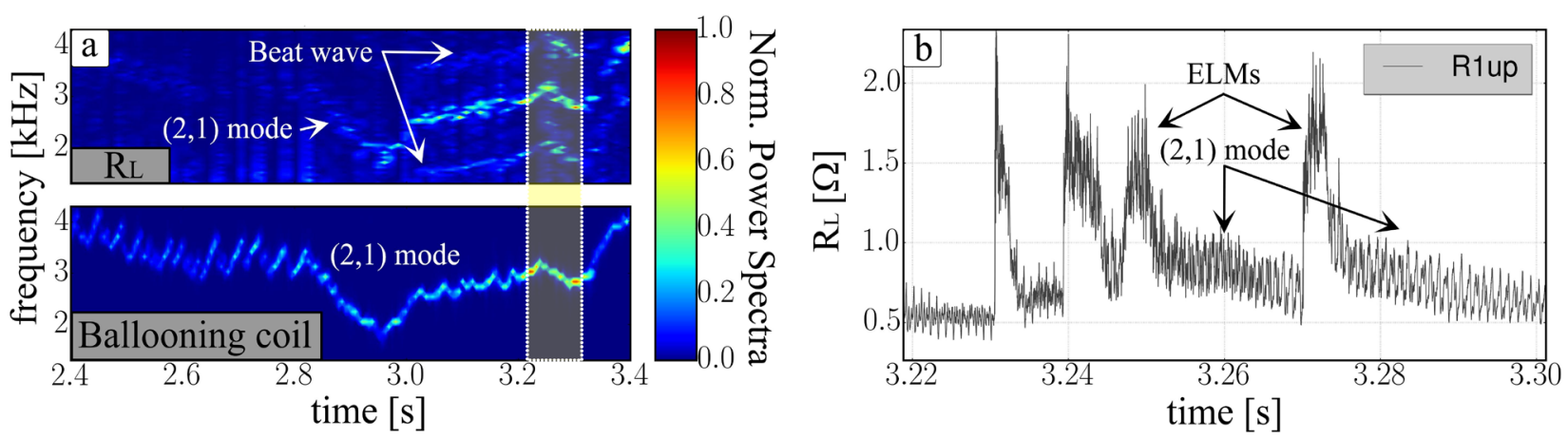

Figure 5. Discharge \#32749. (a) Spectrograms of the loading resistance measured in the upper feeder of a1 and Ballooning coil for reference. The signature of an MHD mode is clearly visible on both signals. (b) Loading resistance during the highlighted time region.

\section{Ion cyclotron fast wave coupling under MHD modes}

MHD modes can also deform the plasma boundary in a non-axisymmetric fashion. The displacement they produce depends upon their radial profile $(\vec{\xi}(r))$ and growth rates $(\gamma)$, supposing that the mode structure can be described as $\vec{\xi}(r, \theta, \phi, t)=\vec{\xi}(r) \cos (m \theta-n \phi+w t) e^{\gamma t}$. We present here the case of a coherent modulation of the loading resistance in the ion cyclotron antennas with the rotation of a $(2,1)$ mode. A spectrogram shows the same frequency components for the loading resistance measurements and a Ballooning coil, which registers the change of radial magnetic field due to the mode rotation (figure 5).

The change in loading resistance is hypothesized to be connected with a density modification in front of the ICRH antennas, due to the radial profile of the MHD mode. The loading resistance spectrogram in figure $\mathbf{5}$ also displays a beat wave pattern from $3 \mathrm{~s}$ on. The reason is the activation of the 3-strap antennas, which are shifted by $1 \mathrm{kHz}$ in their operational frequency with respect to the 2-strap antennas. The $(2,1)$ mode numbers are identified via the poloidal and toroidal arrays of Mirnov and Ballooning coils present on the tokamak vessel.

A loading resistance oscillation an order of magnitude faster (few $\mathrm{kHz}$ ) than the ELM cycle is observed during the appearance of this $(2,1)$ mode, as seen in figure 5 . For the ASDEX Upgrade case, the hybrid $3 \mathrm{~dB}$ couplers close to the RF generators assure a steady load-resilient operation.

\section{Conclusions and outlook}

MP fields induce a LFS midplane non-axisymmetric plasma kink response that influences ion cyclotron antennas coupling properties. Rigid rotation of the MP field causes a harmonic oscillation of the antenna loading resistance. In this study, with relatively fast rotating MP fields $(v=6 \mathrm{~Hz})$ and $I_{\text {coil }}=6.25 \mathrm{kAt}$, the loading resistance is found to change as much as $\Delta R_{L^{\sim}} 0.3 \Omega$. This loading variation accounts for $\sim 20 \%$ of the base value. The $3 \mathrm{~dB}$ hybrid system is able to assure normal operation of the ICRH system. Nevertheless, loading resistance oscillations such as the ones presented in this study need to be taken into account, especially, for future ion cyclotron systems. Higher loading oscillations could be encountered if the plasma displacement becomes larger (i.e. slow rotating MP field with higher coil currents). In future reactors such as ITER, this effect should be considered if MP fields are to be used for ELM control.

MHD modes are found to introduce a coherent loading resistance variation with mode rotation frequency, most likely due to their radial extent. This is illustrated by the specific case of a $(2,1)$ mode. A deeper study is required in order to assess their effects on current and future ion cyclotron systems.

This work has been carried out within the framework of the EUROfusion Consortium and has received funding from the Euratom research and training programme 20142018 under grant agreement No 633053. The views and opinions expressed herein do not necessarily reflect those of the European Commission.

\section{References}

[1] K. Steinmetz, Nuclear Fusion 29, 277 (1989)

[2] R. Bilato, M. Brambilla, D.A. Hartmann, A. Parisot, Nuclear Fusion 45, L5 (2005)

[3] S.P. Hirshmann et. al., Computer Physics Communications, 43, 143 - 155, (1986)

[4] W. Suttrop et. al., Phys. Rev. Lett., 106, 22 - 225004 (2011)

[5] R.A. Moyer et. al., Nuclear Fusion 52, 123019 (2012)

[6] Y. Liu, A. Kirk, Y. Gribov et. al., Nuclear Fusion 51, 8-083002 (2011)

[7] M. Willensdorfer et. al., Plasma Phys. Control Fusion 58, 114004 (2016)

[8] V. Bobkov et al., Nuclear Fusion 53, 093018 (2013)

[9] M. Rott, U. Seidel, B. Streibl et. al., Fusion Engineering and Design 84, 1653-1657 (2009)

[10] P. J. Mc Carthy, Plasma Physics and Controlled Fusion, 54, 015010, (2012) 